\title{
BMJ Open Direct medical costs of type 2 diabetes mellitus in the Philippines: findings from two hospital databases and physician surveys
}

Cecilia Jimeno, ${ }^{1}$ Rosa Allyn Sy, ${ }^{2,3}$ Pepito De La Pena, ${ }^{4}$ Chritopher Cipriano, ${ }^{3}$ Rima Tan, ${ }^{5}$ Araceli Panelo, ${ }^{6}$ Junice Yi Siu Ng (D) ${ }^{7}$

To cite: Jimeno C, Sy RA, De La Pena P, et al. Direct medical costs of type 2 diabetes mellitus in the Philippines: findings from two hospital databases and physician surveys. BMJ Open 2021;11:e049737. doi:10.1136/ bmjopen-2021-049737

- Prepublication history and additional supplemental material for this paper are available online. To view these files, please visit the journal online (http://dx.doi.org/10.1136/ bmjopen-2021-049737).

Received 05 February 2021 Accepted 24 August 2021
Check for updates

(C) Author(s) (or their employer(s)) 2021. Re-use permitted under CC BY-NC. No commercial re-use. See rights and permissions. Published by BMJ.

For numbered affiliations see end of article.

Correspondence to Dr Junice Yi Siu Ng; junice.ng@iqvia.com

\section{ABSTRACT}

Objective To estimate the annual direct medical cost of type 2 diabetes mellitus (T2DM) in hospitals and outpatient care clinics from a healthcare payer perspective in the Philippines.

Design and participants (1) A review of electronic hospital records of people with T2DM in two tertiary hospitals-0spital ng Makati (OsMak) and National Kidney and Transplant Institute (NKTI) and (2) a cross-sectional survey with 50 physicians providing outpatient care for people with T2DM.

Setting Primary, secondary and tertiary healthcare facilities in Metro Manila.

Outcome measures Cost of managing T2DM and its related complications in US dollars (USD) in 2016.

Results A total of 1023 and 1378 people were identified in 0sMak and NKTI, with a complication rate of $66 \%$ and $74 \%$, respectively. In both institutions, the average annual cost per person was higher if individuals were diagnosed with any complication (NKTI: US\$3226 vs US\$2242 and OsMak: US\$621 vs US\$127). Poor diabetes control was estimated to incur higher per person cost than good control in both public outpatient care (poor control, range: US $\$ 727$ to US $\$ 2463$ vs good control, range: US\$614 to US\$1520) and private outpatient care (poor control, range: US\$848 to US\$2507 vs good control, range: US\$807 to US\$1603).

Conclusion The results highlight the high direct medical cost resulting from poor diabetes control and the opportunity for cost reduction by improving control and preventing its complications.

\section{INTRODUCTION}

The number of individuals with diabetes is on the rise in the Philippines, as like across the globe. The International Diabetes Federation estimated 600000 more individuals with diabetes in 2019 than a decade ago, bringing the total estimated number to 4 million in 2019. ${ }^{12}$ This estimated number excluded the 2.6million people who were unaware that they have diabetes. ${ }^{2}$ In a cohort derived from a national nutrition survey, $16 \%$ people developed type 2 diabetes mellitus (T2DM) after a
Strengths and limitations of this study

This is the first costing study on type 2 diabetes in the Philippines using hospital databases.

- This study has identified potential data sources for future costing research.

- The costs presented in this study could be underestimated.

follow-up period of 9 years. ${ }^{3}$ T2DM is a chronic condition that requires continuing medical care. If the condition is well-controlled, it could prevent serious complications, such as kidney failure, and this would lead to cost savings for healthcare systems, payers and individuals. The global economic burden of diabetes stands at US\$1.31 trillion. ${ }^{4}$ Higher costs have been associated with the management of people with T2DM-related complications than those without complications. ${ }^{5-8}$ According to the Philippine Renal Disease Registry Annual Report in 2015, diabetes is the leading cause of kidney failure at $49 \% .^{9}$ Adequate dialysis costs between Philippine pesos (PHP) 300000 to PHP 552000 (equivalent to the US $\$ 5880$ to US $\$ 10820$ ) annually, well beyond the average household income of PHP 267000 (US\$5234). ${ }^{10}$

Given the substantial economic impact of diabetes and its sequelae on individuals and the healthcare system supporting them, detailed costing estimates are necessary to understand costs and resources critical to healthcare system planning. Current cost estimates in the Philippines are limited to data from a cross-sectional survey of 359 respondents recruited from varying levels of healthcare facilities in 2008. The costs of oral medications, insulin and transportation were US $\$ 13 /$ month, US $\$ 20 /$ month and US $\$ 1 /$ visit, respectively. Other direct medical costs, 
such as laboratory tests and procedures, were not part of the research scope. ${ }^{11}$

\section{Healthcare in the Philippines}

Healthcare is financed by a mix of national health insurance, known as PhilHealth, and private out-of-pocket payments, with the latter being the main source of healthcare financing. ${ }^{12} 13$ Coverage by PhilHealth differs by the type of membership. The core benefits include up to 90 days of inpatient care per family, disease-specific outpatient packages and Z-benefits package for a set of catastrophic conditions, including common consequences of diabetes, such as coronary artery bypass, prosthesis and kidney transplants. ${ }^{14}$ These benefits can be accessed at any PhilHealth accredited facility in the country, either government-owned or privately-owned. The Primary Care Benefit package, which includes the provision of diagnostic examinations and provision of medicines for chronic diseases, such as T2DM and hypertension, offered by PhilHealth is only available for indigent and sponsored members. The provision of healthcare is fragmented yet dynamic. ${ }^{13}$ There is a referral system but there is no gatekeeper. Multimorbidity requires contacts with different clinicians, departments or even hospitals. Patients are free to move between facilities if their health improves or declines, or if they desire a second opinion. With the dynamic care pathway and flexible choice of care in different settings, the care may yield significant cost differences. However, there is no single database that aggregates information from all hospitals and primary care. Therefore, a combination of methods had to be used to estimate the cost of services at different facilities. The current study aimed to perform a comprehensive analysis to estimate the direct medical costs of T2DM and its related complications in hospitals and outpatient care clinics from a healthcare payer perspective, that is, all costs of health services provided to patients.

\section{METHODS}

The study methodology has been published. ${ }^{15}$ Briefly, the estimation of direct medical cost of T2DM involved two parts: (1) a retrospective cohort study design using electronic records from two hospitals and (2) a crosssectional survey with 50 physicians. The first part was used to estimate the direct medical costs of people with T2DM-related complications, and the latter was aimed to describe the management cost for those without complications and were routinely followed up at outpatient care.

\section{Retrospective chart review}

Electronic records of people with T2DM who visited Ospital ng Makati (OsMak) and National Kidney and Transplant Institute (NKTI) in 2016 were extracted.

\section{OsMak}

OsMak is a 300-bed tertiary government general hospital based in Makati City, mainly serving its city residents.
Direct medical costs are obtained from the accounting database of OsMak and were traceable to laboratory tests, procedures, haemodialysis, professional fees, room and board and medications during an inpatient stay, as well as procedures and haemodialysis during an outpatient visit (as shown in online supplemental figure 1). Professional fees were chargeable if patients were not a resident of Makati City. In-house laboratory tests were only performed for some outpatients, while the rest were tested in private laboratories, and in which case, the costs were not included in this study. Maintenance medications for outpatients were dispensed in retail pharmacies. Data for the maintenance medications were only captured electronically starting 2018 and hence, were not available at this point in the study.

\section{NKTI}

The NTKI is a specialised hospital and the only national referral centre for patients with kidney disease situated in Quezon City. The accounting database of NKTI included costs of laboratory tests, procedures, haemodialysis, professional fees, room and board, medications incurred by both public and private inpatients, laboratory tests, procedures, haemodialysis and professional fees for public outpatients (as shown in figure 1). Records of private outpatients were not captured. It was not possible to differentiate public and private inpatients, and only 82 of the 386 beds were allotted to public patients. Similar to OsMak, outpatient prescriptions were filled in retail pharmacies and were not captured in the hospital databases.

Online supplemental appendix 1 further describes the cost structure in each hospital.

\section{Eligibility}

The study included individuals with T2DM who were aged 18 or above. T2DM was identified using the 10th revision of the International Statistical Classification of Diseases (ICD-10) code E11 from the database of NKTI. Keywords, such as 'T2D' and 'Type 2 diabetes', were used to search for people with T2DM from the free-text diagnosis in the database of OsMak. Those prescribed with oral glucoselowering therapy were also included. This data extraction was conducted by two independent coders using different algorithms. A reviewer reconciled the differences in coding.

\section{Identification of T2DM complications}

The Diabetes Severity Complications Index (DSCI) includes seven categories of complications: retinopathy, nephropathy, neuropathy, cerebrovascular disease, cardiovascular disease, peripheral vascular disease and metabolic disease. Each category comprises a list of conditions and their corresponding ICD-10 codes. Similar to the determination of T2DM, ICD-10 codes and specific keywords were used to identify T2DM-related complications. In this study, the DSCI was solely used to provide a reference list of complications. No scores were generated to model the severity of its complications. 


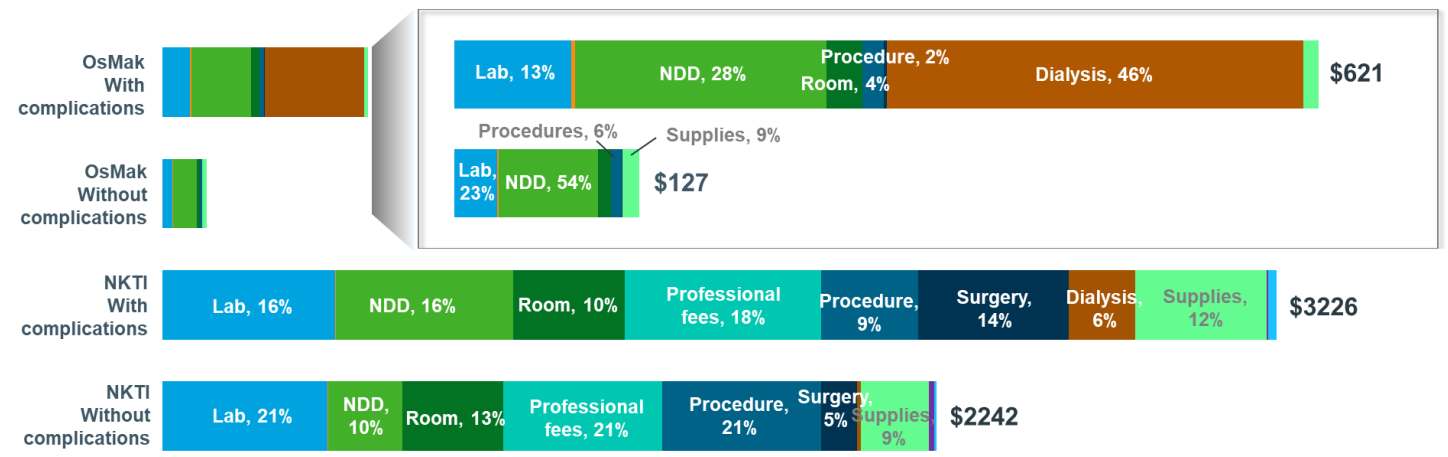

$$
\begin{aligned}
& \text { - Laboratory (Lab) } \\
& \text { - Room } \\
& \text { - Hemodialysis }
\end{aligned}
$$

- Oral anti-diabetics - Professional fe - Supplies/others
- Insulin

- Procedure - Palliative
- Surgery/Minor OT
- Non-diabetes-related drugs (NDD

Figure 1 Breakdown of costs in US dollars (\$) in OsMak and NKTI in 2016, by presence of complications. NKTI, National Kidney and Transplant Institute; OsMak, Ospital ng Makati.

\section{Physician interviews \\ Eligibility}

A total of 50 physicians practicing in internal medicine, family medicine, diabetology or endocrinology were interviewed in from February to August 2020. The sample size was determined to achieve maximum variation sampling, in terms of type of facility (hospital, primary care clinic and specialist clinic), discipline (endocrinology, diabetology, family medicine and internal medicine) and geographical location (Makati City and Quezon City). The sampling distribution is found in online supplemental appendix 2. They were selected from a list of physicians who had registered interest in participating in market research with a contract research organisation. These physicians had at least 8 years of experience providing care for people with T2DM and a self-reported strong or moderate knowledge of the costs of the clinic or institution's services. Each month, they must also be treating, on average, 10 or more cases of hypoglycaemia among people with T2DM.

\section{Data collection}

The resource utilisation and costs for diabetes control in outpatient care were obtained over two rounds of data collection, punctuated by a consultation with a group of study advisors, who were also the coauthors of this study. The first round involved 26 physicians equally split between Makati City and Quezon City. The survey comprised open-ended questions on resource utilisation pertaining to prescription regimens, doctor's consultations and diagnostics and monitoring tests and unit cost of the resources. The study advisors discussed the aggregate results obtained from the first round and the survey questions and approach for the second round. The second survey, administered to another group of 24 physicians in Makati City and Quezon City, aimed to assess the respondents' agreement to the aggregate responses obtained from the first round. A sample of the survey questions can be found in online supplemental appendix 3. Respondents who did not agree to the findings were asked to indicate the values, either proportions or frequencies, in the context of their practice.

\section{Interviews}

Structured interviews, each lasting for about $45 \mathrm{~min}$, were conducted in English. The first round was conducted face-to-face in the respondents' offices, while the second round was conducted online. Physicians were asked to state the typical resources needed to manage hypoglycaemia, defined as blood sugar level below $70 \mathrm{mg} / \mathrm{dL}$, as well as four groups of people with T2DM and different comorbidities of interest: (1) hypertension only, (2) dyslipidaemia only, (3) hypertension and dyslipidaemia and (4) no comorbidities of interest. Hypertension and dyslipidaemia were the most common comorbidities found among people with T2DM in the Philippines. ${ }^{16}$ The resources included prescription regimens, doctor consultations and diagnostic and monitoring tests. Unit costs of treatment and tests were obtained from the administrator if the physicians could not provide the cost.

\section{Patient and public involvement}

No patient was involved in this study.

\section{Statistical analysis}

Descriptive analysis, without any test of significance, was conducted using Stata V.15. All costs were standardised to the US dollars in 2016 (US\$ $1 \approx$ PHP 47.492). An annual discount rate of $3 \%$ was applied to the costs estimated from the physician interviews.

\section{RESULTS}

\section{Retrospective chart review}

Patient characteristics

Table 1 lists the demographic and clinical characteristics of the people with diagnosed T2DM at OsMak and NKTI. There were 1023 people in OsMak, of which $40 \%$ were men. NKTI treated 1378 people with T2DM, and $55 \%$ were men. The mean (SD) age was lower for persons seeking 


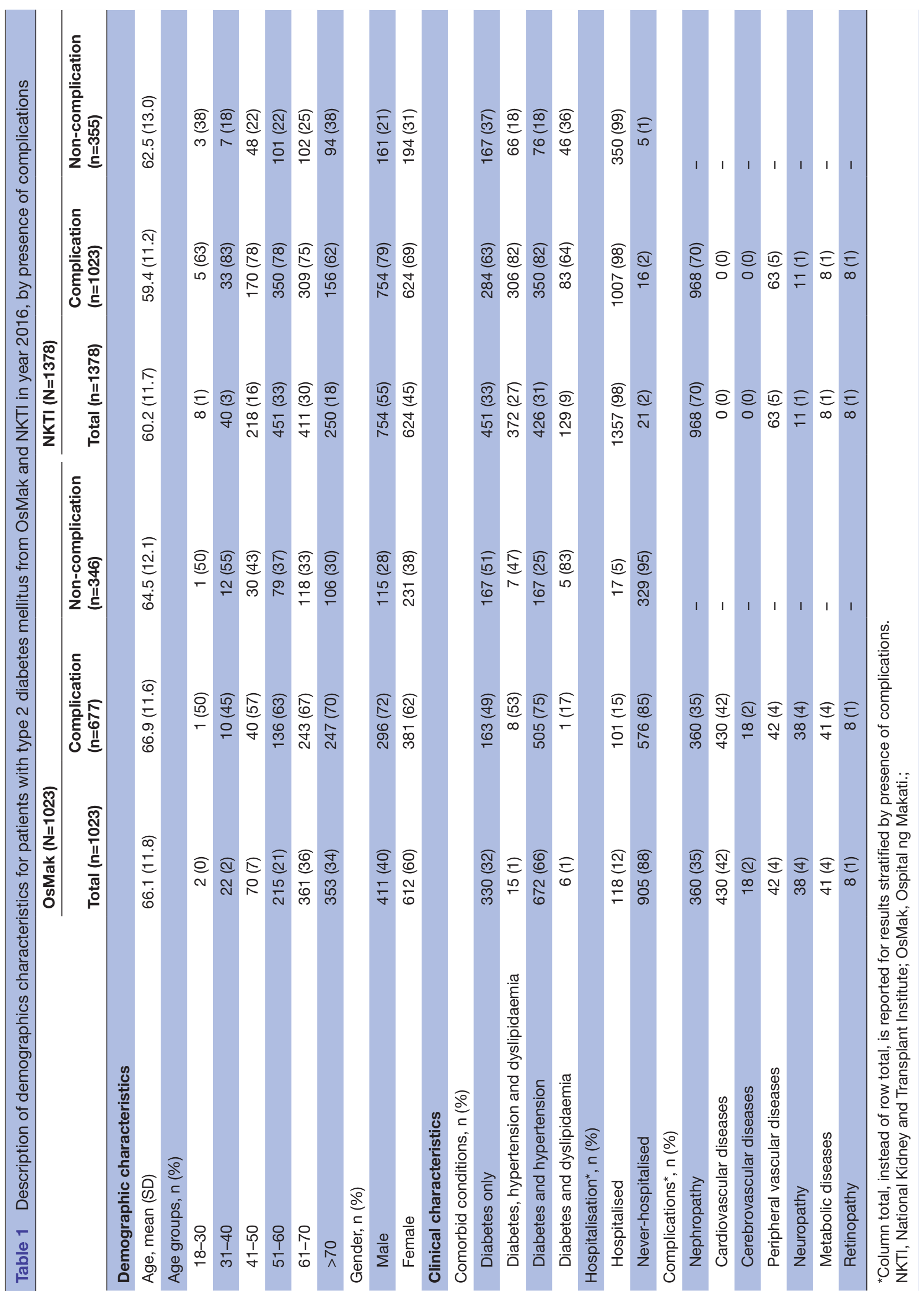


care in NKTI at 60.2 years (11.7 years) than OsMak at 66.1 years (11.8 years). Hypertension (OsMak: $67 \%$ and NKTI: $58 \%$ ) was a more common comorbidity compared with dyslipidaemia (OsMak: $2 \%$ and NKTI: $36 \%$ ) in both institutions. Diabetes-related complications were found in $66 \%$ and $74 \%$ of the people at OsMak and NKTI, respectively. Cardiovascular diseases $(42 \%)$ were the most prevalent complication in OsMak, while nephropathy was the most common complication in NKTI, occurring in $70 \%$ of the individuals. People with diabetes-related complications in OsMak were found to be older, male and had a higher likelihood of being diagnosed with hypertension. Similarly, in NKTI, the prevalence of complications was higher among older, male individuals with hypertension and dyslipidaemia.

Healthcare resource utilisation

For both institutions, the presence of complications was associated with a higher propensity of being hospitalised and longer length of stay. Around 15\% of the individuals with complications in OsMak were hospitalised compared with $5 \%$ of those without complications. The median (IQR) number of days of hospital stay was 7 (3.5-13) days for people with complications versus 5 (3.5-13) days for those without complications. In NKTI, almost all (98\%) were hospitalised, but the presence of complications also prolonged the median (IQR) length of stay (6 (3-10) days vs 4 (2-7) days).

\section{Healthcare cost}

The annual mean (SD) cost was much higher in NKTI (US\$2973 (US\$6166)) than in OsMak (US\$454 (US\$1253)). In both institutions, the average annual cost per person was higher if individuals were diagnosed with any complication (figure 1). At OsMak, the mean (SD) cost of managing people with complications (US\$621 (US\$1487)) was almost five times greater than those without complications (US\$127 (US\$398)). The impact of diabetes-related complications in annual mean (SD) cost per person was less pronounced for individuals at NKTI (US\$3226 (US\$6749) vs US\$2242 (US\$3957)). The relationship between medical cost and complications was similar on stratification by hospitalisation status. The average (SD) costs among individuals who were hospitalised were higher for those with complications (US\$1909 (US\$2099)), compared with those without complications (US\$1203 (US\$1400)), as shown in table 2. This was also the case for never-hospitalised individuals (US\$395 (US\$1221) vs US\$72 (US\$86)). In NKTI, people with complications also incurred higher costs regardless of hospitalisation status (hospitalised: US\$3272 (\$6792) vs US\$2270 (US\$3978) and never-hospitalised: US\$351 (US\$211) vs US\$273 (US\$101)), as shown in table 3.

At OsMak, the main cost drivers for individuals identified with complications were dialysis $(46 \%)$, non-diabetesrelated drugs $(28 \%)$, laboratory tests $(13 \%)$ and room and board or emergency fee (4\%) (figure 1). Costs of oral glucose-lowering agents $(0.01 \%)$ and insulin $(0.4 \%)$ were minimal. In contrast, non-diabetes-related drugs (54\%) represented the biggest component of the annual mean cost for people without complications, followed by laboratory tests $(23 \%)$, supplies $(9 \%)$ and procedures $(6 \%)$. Oral glucose-lowering agents and insulin constituted $0.003 \%$ and $1 \%$ of the total cost, respectively. Among those who were hospitalised, $61 \%$ and $65 \%$ of people with

Table 2 Description of direct cost in US\$ for patients with type 2 diabetes mellitus from OsMak in year 2016, by presence of complications

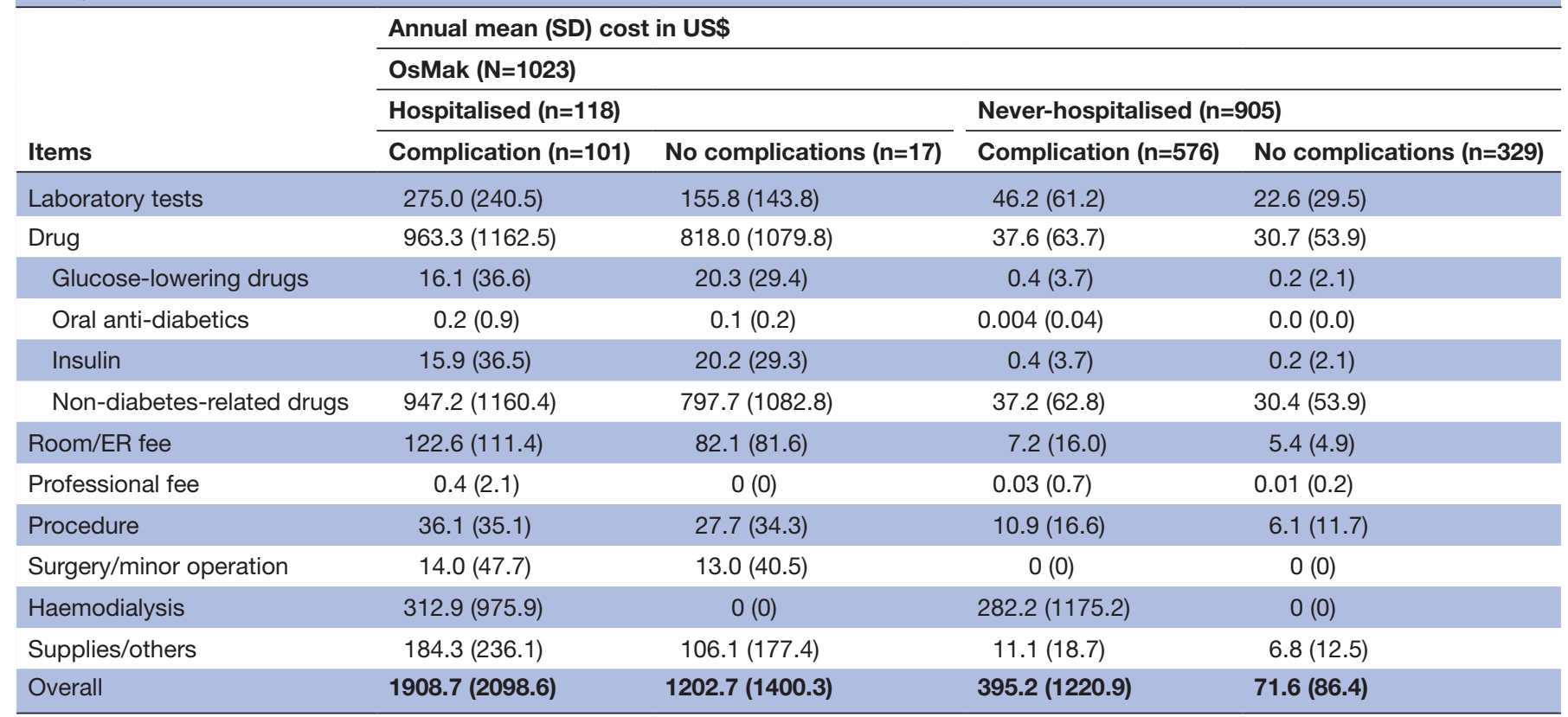

ER, emergency room; OsMak, Ospital ng Makati. 
Table 3 Description of direct cost in US\$ for patients with type 2 diabetes mellitus from NKTI in year 2016, by presence of complications

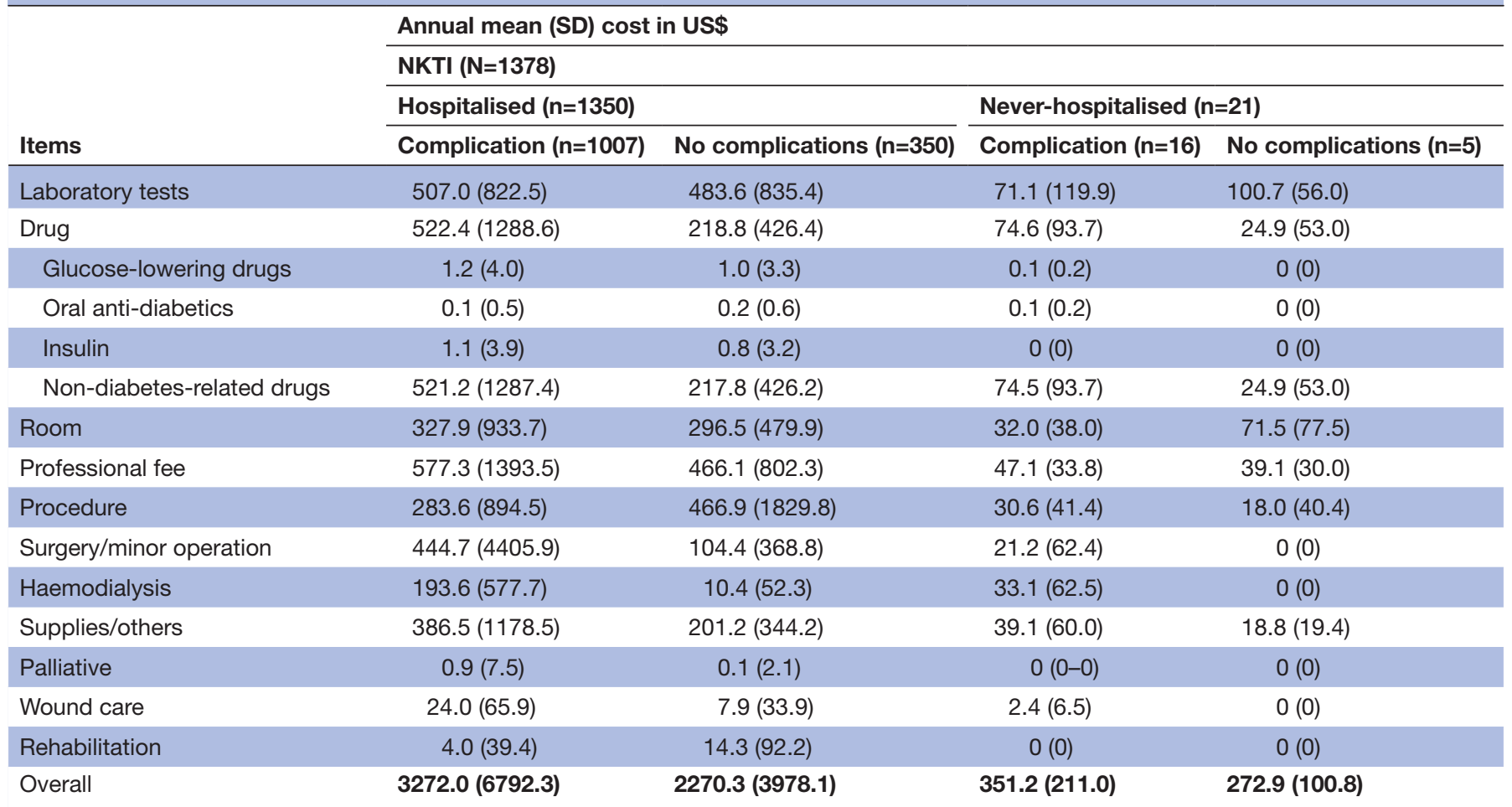

NKTI, National Kidney and Transplant Institute.

and without complications, respectively, received glucoselowering agents, as shown in online supplemental table 1. Almost all individuals were prescribed medications to manage conditions other than T2DM (complications: 98\% and non-complications: $100 \%$ ). The average annual costs (SD) of the glucose-lowering agents amounted to US\$26 (US\$44) and US\$31 (US\$32) for those with complications and without complications, respectively, while the mean (SD) costs for non-diabetes-related drugs were US\$966 (US\$1164) and US\$798 (US\$1083). Among the $110(11 \%)$ people who underwent haemodialysis, the mean (SD) total medical cost incurred in 2016 was US\$1764 (US\$2749) (not shown in table).

Professional fees (18\%), non-diabetes-related drugs $(16 \%)$, laboratory tests $(16 \%)$ and surgery $(14 \%)$ were the key cost components for people with diabetes-related complications at NKTI (figure 1 ). The costs of oral glucoselowering agents $(0.003 \%)$ and insulin $(0.3 \%)$ were negligible. Among those without complications, professional fees, procedures, laboratory tests (each 21\%) and room and board (13\%) accounted for the majority of the costs. Costs of oral glucose-lowering drugs $(0.007 \%)$ and insulin $(0.4 \%)$ were minimal. Only a quarter $(26 \%)$ of the hospitalised individuals had been prescribed glucose-lowering drugs (complications: $23 \%$ and non-complications: $35 \%$ ), as shown in online supplemental table 2. Those with complications incurred a similar mean (SD) cost of US $\$ 5$ (US\$7) as those without complications at US\$3 (US\$5). Almost all individuals received non-diabetes-related drugs (complications: 99\% and non-complications: 96\%), and its average costs were doubled in the presence of complications (US\$526 (\$1292) vs US $\$ 228$ (US\$433)). A total of $592(43 \%)$ people who underwent haemodialysis in NKTI incurred an average (SD) of US\$3489 (US\$5516) in total medical cost in 2016 (not shown in table).

\section{Physician interviews}

An outpatient receiving public care with poor T2DM control (range: US\$727 to US\$2463 per person per year) incurred a higher cost than those with good control (range: US $\$ 614$ to US $\$ 1520$ per person per year), as illustrated in figure 2. These ranges apply to patients of different disease groups: no comorbidities (ie, hypertension and dyslipidaemia), one comorbidity or both comorbidities. A similar trend was also seen in outpatients attending private facilities (poor control, range: US $\$ 848$ to US\$2507 per person per year and good control, range: US $\$ 807$ to US $\$ 1603$ per person per year). People with more comorbidities had higher expenditure. Generally, private patients also incurred higher annual medical costs than public patients. The most commonly prescribed medication regimens for people with T2DM and comorbidities are shown in online supplemental table 3 . The unit costs are found in online supplemental table 4 . The median number of hypoglycaemic events per person per year was approximated to be 8 for government facilities and 13 for private facilities. The average cost of managing a hypoglycaemic event in public and private facilities 
(a) Annual cost per person incurred in public health facilities

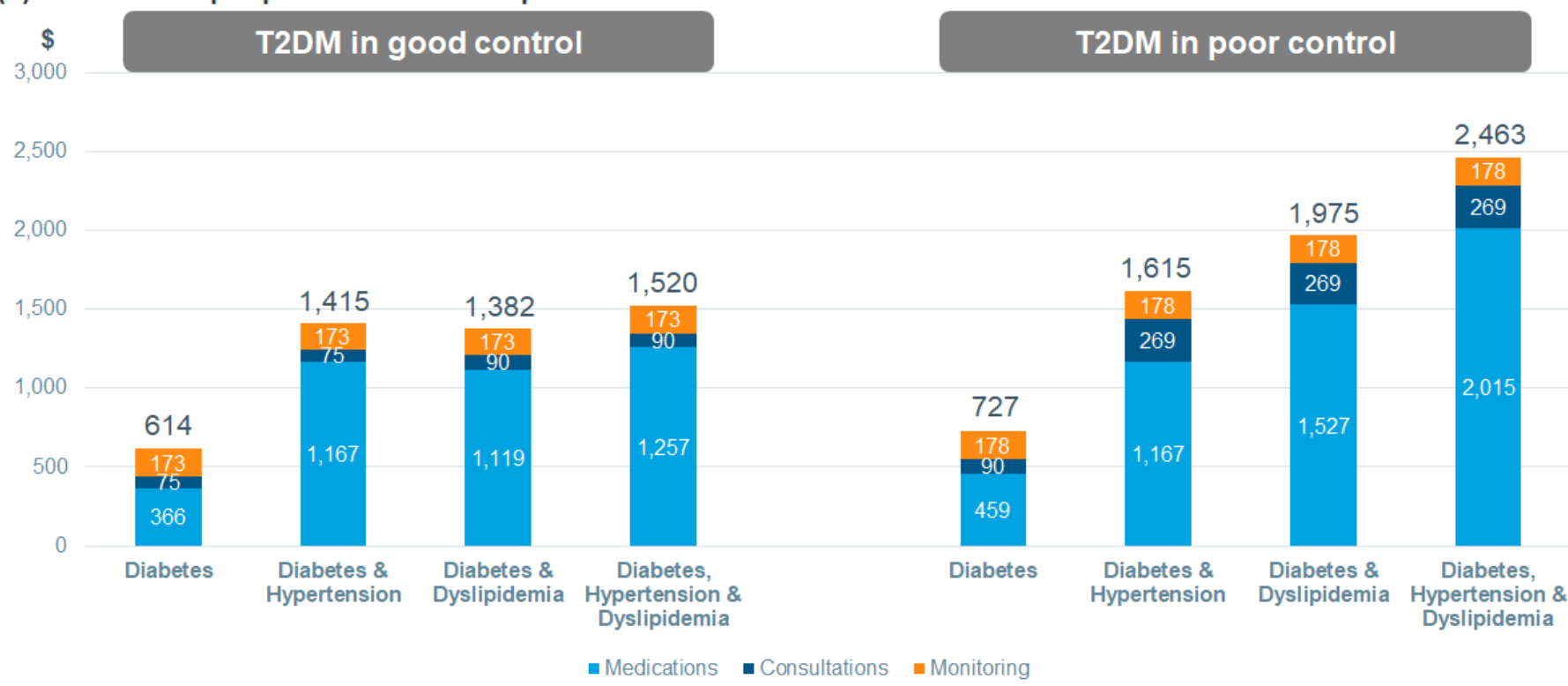

(b) Annual cost per person incurred in private health facilities

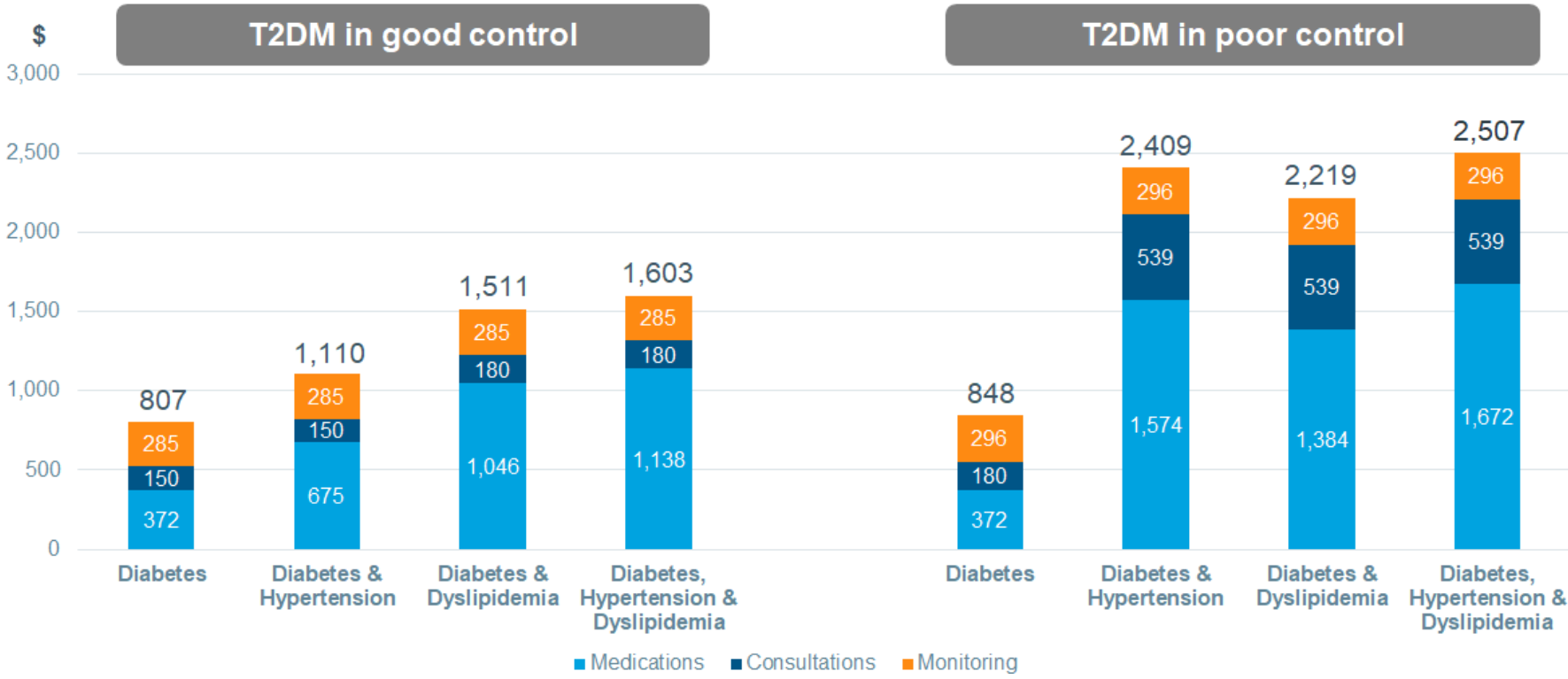

Figure 2 Breakdown of estimated annual cost per person in US dollars (\$) in (A) public and (B) private outpatient setting in 2016, by presence of complications. (A) Annual cost per person incurred in public health facilities. (B) Annual cost per person incurred in private health facilities. T2DM, type 2 diabetes mellitus.

was around US\$35 (comprising US\$32 for diagnosis and US\$3 for treatment) and US\$93 (comprising US\$90 for diagnosis and US\$4 for treatment), respectively.

\section{DISCUSSION}

\section{Principal findings}

This study aimed to estimate the costs of managing diabetes in primary care through interviews with physicians at clinics and tertiary care through the use of hospital database. Our findings revealed a high cost needed to manage people with T2DM in the Philippines. It also showed that resource utilisation and treatment cost were substantially higher for people with complications in the hospital setting, whether or not they are hospitalised. Only $12 \%$ of the individuals with T2DM seeking care in OsMak were hospitalised, in contrast with the $98 \%$ of people hospitalised in NKTI. This difference is attributable to the institution type-the former provides general and specialised care, and the latter provides only specialised care. Based on the physician interviews, people with T2DM incurred high outpatient costs, especially those who have poor control of T2DM.

\section{Comparison with other studies}

Our findings are consistent with studies examining the associations between diabetes-related complications and economic burden of T2DM in Asia. In a hospital-based 
study in Thailand, the median cost for those with complications was four times higher than those without complications. ${ }^{17}$ A retrospective claim database analysis in Taiwan found that patients with diabetes-related complications had higher resource utilisation patterns and costs, namely hospitalisation, outpatient visits and emergency room visits. ${ }^{5}$ Economic burden was also intensified for people with complications in a similar retrospective database study conducted in Vietnam, in which $70 \%$ of the total direct medical costs were attributed to diabetesrelated complications. ${ }^{18}$

\section{Clinical relevance and policy implications}

To our knowledge, this study is the first to estimate the costs incurred in the control of T2DM in hospital and outpatient settings in the Philippines. The findings could be useful for policymakers in designing appropriate strategies for diabetes control. First, the high per capita spending needed to manage individuals with diabetes revealed that the introduction of a multilevel health promotion strategy could improve current diabetes control. Evidence-based community interventions, such as the volunteer-led Community Health Assessment Program, could focus on diabetes education and prevention. ${ }^{19}$ At the healthcare level, regular screening of diabetes could facilitate prompt diagnosis at primary care level, leading to people developing fewer complications. The upcoming implementation of the universal healthcare policy may also improve access to care at the primary healthcare level ${ }^{20}$ if sufficient reimbursement is provided for individuals with T2DM to (even partially) cover outof-pocket costs, such as monitoring or medication costs. Second, our findings showed the costly consequence of managing diabetes complications, which could be averted by improving current management. Expanding the arsenal of glucose-lowering drugs in the formularycurrently limited to metformin, gliclazide and human insulin-could help to prevent downstream risks of complications. For instance, most guidelines now suggest evaluating atherosclerotic cardiovascular disease or chronic kidney disease in people with T2DM and initiate glucagon-like peptide-1 receptor agonists or sodiumglucose co-transporter-2 inhibitors that have the potential to prevent or delay cardiovascular and renal diseases. ${ }^{21}$ Next, insulin therapy is required when lifestyle modifications and oral glucose-lowering agents failed to provide adequate metabolic control..$^{22}$ However, despite the wide availability of insulin and its inclusion in the formulary, through the physician interviews in this study, we found an under-utilisation of insulin, especially in public health facilities. Under the Department of Health Insulin Medicines Access Program, insulin is provided at no cost to individuals who require it, as part of the effort to provide quality essential medicines defined by the WHO. ${ }^{23}{ }^{24}$ This programme also entailed a one-time training for health workers on the effective use of insulins. Nonetheless, ongoing and regular training could reinforce knowledge and skills, improving their confidence in administering insulin. Taken together, these measures could significantly increase access to healthcare and medications with reduced overall expenditures.

\section{Strengths and limitations}

The costs presented in this study were underestimated because of the following factors. First, since healthcare is a free system, individuals could go to any medical facility for other specialised care. As such, the hospital databases were unable to encapsulate the entire patient journey. This warrants further research using an integrated database to provide a comprehensive view of the control and cost of diseases. The PhilHealth database was unsuitable as it contained bundled costs and mainly inpatient data without complete secondary diagnosis codes. ${ }^{25}$ We also attempted to include data from St Luke's Medical Center, the largest private hospital in the Philippines but were met with little success as some data variables, such as secondary diagnoses, were not captured digitally. Hence, the results did not reflect the cost incurred by people receiving care in a private healthcare setting. In the Philippines, around $65 \%$ of the hospitals are privately-owned. ${ }^{26}$ Second, although the cost of outpatient care was estimated through physician interviews, it is still an underestimation as it did not include the medications needed to manage T2DM-related complications. In estimating the cost per hypoglycaemic event, we did not include consultation costs due to the variability in the diagnostic workup. There could also be under-reporting of the resources needed to manage hypoglycaemia since the study sample did not include emergency physicians. Next, the cost of medications estimated using the database of OsMak did not include the consultation fees for most outpatients and the cost of maintenance medications provided in the retail pharmacies, both of which were funded by the City of Makati government. NKTI, a government-subsidised hospital, procured drugs at low prices and charged the same for both public and private patients. This was an unlikely occurrence for private patients in other public or private health facilities. Furthermore, this study did not capture individuals with T2DM and cardiovascular or cerebrovascular diseases in NKTI, as the information was stored in a separate database. Excluding the management of these diseases would underestimate the cost reported in this study. The census data in NKTI in 2016 reported that $62 \%$, $7 \%$ and $6 \%$ of the people with T2DM were also diagnosed with coronary artery disease, congestive heart failure (CHF) and acute coronary syndrome, respectively. ${ }^{27}$ In a separate study, the average medical cost of CHF hospitalisation in 2014 was estimated to range from US $\$ 436$ to US $\$ 636$ per person in government hospitals, and it was US\$639 to US\$941 in private hospitals. ${ }^{28}$ The cost of haemodialysis estimated in this study was also lower than expected. A typical individual would usually need dialysis three times a week, totalling 156 sessions a year. PhilHealth covers only 90 of these sessions. With each session costing around US\$53, each person would potentially have to pay around US\$3498 in a year, which was almost equivalent to, if not higher than, the Gross Domestic Product (GDP) per capita of US\$2941. The lower cost of haemodialysis reported in this study could be explained by 
the possibility of individuals receiving dialysis outside of NKTI or receiving coverage from charity organisations. Even so, in 2016, PhilHealth spent a total of US $\$ 170$ million on haemodialysis, which accounts for $8 \%$ of the total benefit payment in the same year, compared with $6 \%$ (US\$105million) in 2014. This proportion was increased to $9 \%$ of the total costs of US $\$ 207$ million in $2018 .{ }^{29}$ With diabetes being the biggest driver of kidney failure in the Philippines, the importance of prevention and control of T2DM becomes even greater. This study should also be interpreted in view of the following limitations. As cross-sectional data were used, we are unable to draw the causal relationship between medical expenditures and T2DM, and how the insurance or residence status or public or private care impact this relationship. Furthermore, most data were collected from Metro Manila and may not be generalisable to other areas in the Philippines. Finally, the results were limited by the small sample size of physicians surveyed. Additional analyses in a larger population are required to validate our study findings.

This study had a few strengths. It used electronic databases instead of collecting information based on recall, as with the previous literature. ${ }^{11}$ These databases were the actual payments borne by the medical care providers. The outpatient costs estimated using physician interviews also complemented the findings obtained from both hospitals, which did not capture the cost of maintenance medications for outpatients.

\section{Conclusion}

This study provides previously unavailable information in the Philippines on the high direct medical costs in the control of T2DM borne by the healthcare system and the higher healthcare costs observed among people with T2DM-related complications (NKTI: US\$3226 vs US $\$ 2242$ and OsMak: US $\$ 621$ vs US $\$ 127)$. Poor diabetes control was also associated with higher cost of outpatient care (poor control, range: US $\$ 727$ to US $\$ 2507$ vs good control, range: US\$614 to US\$1603). Improving the control of T2DM should not be limited to specialist care but be promptly initiated at the primary care level, thereby reducing morbidity and mortality and subsequently lowering the economic and clinical burden exerted by diabetes on its healthcare system, but also the cost borne by the individuals with T2DM. This study estimated separately the direct medical costs of managing T2DM in hospitals and outpatient clinics. Future studies could examine the direct medical costs of the entire patient journey in the healthcare system.

\section{Author affiliations}

${ }^{1}$ Department of Pharmacology and Toxicology, and Section of Endocrinology, Diabetes and Metabolism, University of the Philippines Manila College of Medicine, Manila, Metro Manila, Philippines

${ }^{2}$ Section of Endocrinology, Diabetes, Metabolism and Nutrition, Ospital ng Makati, Makati, Metro Manila, Philippines

${ }^{3}$ Research Development and Innovation, Ospital ng Makati, Makati City, Philippines ${ }^{4}$ Division of Internal Medicine, National Kidney and Transplant Institute, Quezon City, National Capital Region, Philippines

${ }^{5}$ Institute for Studies on Diabetes Foundation Inc, Marikina City, Metro Manila, Philippines
${ }^{6}$ Department of Medicine, University of the East Ramon Magsaysay Memorial Medical Center, Quezon City, Philippines

${ }^{7}$ Real-World Insights, IQVIA Asia Pacific, Singapore

Twitter Cecilia Jimeno @ceciledoc and Chritopher Cipriano @ciprianomd

Acknowledgements We would like to recognise valuable input from Jørgen Kronborg Jungersen, Dr Sagar Anil Chandekar, Dr Praful Chakkarwar, Henrik Bendix Dahl, Dr Ahsan Shoeb, Dr Ma Teresa Dioko, Maija llona Lebel, Erik Wiebols and Sirinthip Petcharapiruch in the conception of this study. We also appreciate Melanie Tobias from National Kidney and Transplant Institute, Florendo Jr Del Rosario from Ospital ng Makati and IQVIA Primary Intelligence (Philippines) for the data collection. We thank Chen Chuang and Miss Tan Wenjing from IQVIA Advanced Analytics Shanghai Hub for providing extensive statistical advice and support.

Contributors CJ and JYSN were involved in the conception and design of the study. JYSN analysed the data. All authors (CJ, RAS, PDLP, CC, RT, AP and JYSN) discussed the results and made a substantial, direct and intellectual contribution to the work. JYSN drafted the manuscript based on the discussion, which was approved by all authors for submission.

Funding This study is funded by Novo Nordisk (2017-06-14T10_04_30) to support the investigators and data collection. This study is conducted by IQVIA Asia-Pacific.

Competing interests JYSN served as a consultant to Novo Nordisk and CJ, RAS, PDLP, CC, RT and AP receive research support from Novo Nordisk.

Patient consent for publication Not required.

Ethics approval This study was approved by the Department of Health Single Joint Research Ethics Board (SJREB-2018-04) and the Cardinal Santos Medical Center Research Ethics Review Committee (2018-035).

Provenance and peer review Not commissioned; externally peer reviewed.

Data availability statement Data are available upon reasonable request. Data may be obtained from a third party and are not publicly available. Data may be obtained from a third party and are not publicly available. The survey data are not publicly available due to ethical restrictions on participant privacy. Data for this study are available upon request to others in the scientific community.

Supplemental material This content has been supplied by the author(s). It has not been vetted by BMJ Publishing Group Limited (BMJ) and may not have been peer-reviewed. Any opinions or recommendations discussed are solely those of the author(s) and are not endorsed by BMJ. BMJ disclaims all liability and responsibility arising from any reliance placed on the content. Where the content includes any translated material, BMJ does not warrant the accuracy and reliability of the translations (including but not limited to local regulations, clinical guidelines, terminology, drug names and drug dosages), and is not responsible for any error and/or omissions arising from translation and adaptation or otherwise.

Open access This is an open access article distributed in accordance with the Creative Commons Attribution Non Commercial (CC BY-NC 4.0) license, which permits others to distribute, remix, adapt, build upon this work non-commercially, and license their derivative works on different terms, provided the original work is properly cited, appropriate credit is given, any changes made indicated, and the use is non-commercial. See: http://creativecommons.org/licenses/by-nc/4.0/.

ORCID iD

Junice Yi Siu Ng http://orcid.org/0000-0003-3072-0386

\section{REFERENCES}

1 IDF diabetes atlas: 4th edn, 2009. Available: https://www.idf.org/ component/attachments/attachments.html?id=811\&task=download [Accessed 12 Nov 2020].

2 Quach H, Prince HM. Clinical practice guideline: multiple myeloma. Australia: Myeloma Australia, 2019.

3 Soria MLB, Sy RG, Vega BS, et al. The incidence of type 2 diabetes mellitus in the Philippines: a 9-year cohort study. Diabetes Res Clin Pract 2009;86:130-3.

4 Bommer C, Heesemann E, Sagalova V, et al. The global economic burden of diabetes in adults aged 20-79 years: a cost-of-illness study. Lancet Diabetes Endocrinol 2017;5:423-30.

5 Cheng S-W, Wang C-Y, Chen J-H, et al. Healthcare costs and utilization of diabetes-related complications in Taiwan: a claims database analysis. Medicine 2018;97:e11602. 
6 Alva ML, Gray A, Mihaylova B, et al. The impact of diabetes-related complications on healthcare costs: new results from the UKPDS (UKPDS 84). Diabet Med 2015;32:459-66.

7 Chen H-L, Hsiao F-Y. Risk of hospitalization and healthcare cost associated with diabetes complication severity index in Taiwan's National health insurance research database. J Diabetes Complications 2014;28:612-6.

8 Zhuo X, Zhang P, Barker L, et al. The lifetime cost of diabetes and its implications for diabetes prevention. Diabetes Care 2014;37:2557-64.

9 NKTI. Philippine renal disease registry: PRDR annual report, renal disease control program (REDCOP). Philippines: National Kidney and Transplant Institute (NKTI) and Epidemiology Bureau of the Department of Health (DOH), 2015.

10 WHO. Noncommunicable diseases (NCD) country profiles. WHO document production services. Geneva, Switzerland: World Health Organization, v. http://www.who.int/nmh/countries/phl_en.pdf

11 Higuchi M. Access to diabetes care and medicines in the Philippines. Asia Pac J Public Health 2010;22:96S-102.

12 Ulep VGT, Dela Cruz NAO. Analysis of out-of-pocket expenditures in the Philippines. J Philipp Dev 2013;40:93.

13 Dayrit MM, Lagrada LP, Picazo OF. The Philippines health system review, 2018

14 PhilHealth. PhilHealth benefits Philippines: Philippine health insurance Corporation. Available: https://www.philhealth.gov.ph/ benefits/\#gsc.tab=0 [Accessed 23 Nov 2020].

$15 \mathrm{Ng}$ JYS, Clement IJ, Jimeno C, et al. Estimating direct medical costs of type 2 diabetes mellitus in the Philippines: a protocol. BMJ Open 2020;10:e025696.

16 Paz-Pacheco E, Jimeno C. Diabetes care in the Philippines. J ASEAN Fed Endocr Soc 2015;30:118-23.

17 Chatterjee S, Riewpaiboon A, Piyauthakit P, et al. Cost of diabetes and its complications in Thailand: a complete picture of economic burden. Health Soc Care Community 2011;19:289-98.

18 Tuan Kiet Pham H, Tuyet Mai Kieu T, Duc Duong T, et al. Direct medical costs of diabetes and its complications in Vietnam: a national health insurance database study. Diabetes Res Clin Pract 2020;162:108051.

19 Agarwal G, Angeles RN, Dolovich L, et al. The community health assessment program in the Philippines (CHAP-P) diabetes health promotion program for low- to middle-income countries: study protocol for a cluster randomized controlled trial. BMC Public Health 2019;19:682.

20 Health Policy Development and Planning Bureau (HPDPB). National objectives for health 2011-2016, health sector reform agenda Monographs. Manila, Philippines, 2012: 5-7.

21 Buse JB, Wexler DJ, Tsapas A, et al. 2019 update to: management of hyperglycemia in type 2 diabetes, 2018. A consensus report by the American diabetes association (ADA) and the European association for the study of diabetes (EASD). Diabetes Care 2020;43:487-93.

22 American Diabetes Association. Pharmacologic approaches to glycemic treatment: Standards of medical care in diabetes-2019. Diabetes Care 2019;42:S90-102.

23 Department of Health. Insuin access program Philippines. Available: https://www.doh.gov.ph/faqs/Insulin-Access-Program [Accessed 23 Nov 2020].

24 World Health Organization. Essential medicines, 2017. Available: https://www.who.int/medicines/services/essmedicines_def/en/ [Accessed 23 Nov 2020].

25 JYS N, Ramadani RV, Hendrawan D. National health insurance databases in Indonesia, Vietnam and the Philippines. Pharmacoecon Open 2019;3:517-26.

26 Health Facilities and Services Regulatory Bureau. List of government and private hospitals. Manila, Philippines: Health Facilities and Services Regulatory Bureau, 2017.

27 NKTI. NKTI Census (Data on record). Philippines: National Kidney and Transplant Institute, 2016.

28 Tumanan-Mendoza BA, Mendoza VL, Bermudez-Delos Santos AAA, et al. Economic burden of hospitalisation for congestive heart failure among adults in the Philippines. Heart Asia 2018;10:e011039.

29 Philhealth stats \& charts, 2018. Available: https://www.philhealth.gov. ph/about_us/statsncharts/snc2018.pdf [Accessed 12 Nov 2020]. 